\title{
LXI. On the gas voltaic battery.-Experiments made with a view of ascertaining the rationale of its action and its application to eudiometry
}

\author{
W.R. Grove Esq. M.A. F.R.S.
}

To cite this article: W.R. Grove Esq. M.A. F.R.S. (1844) LXI. On the gas voltaic battery.-Experiments made with a view of ascertaining the rationale of its action and its application to eudiometry, Philosophical Magazine Series 3, 24:161, 422-432, DOI: $10.1080 / 14786444408644891$

To link to this article: http://dx.doi.org/10.1080/14786444408644891

曲 Published online: 30 Apr 2009.

Submit your article to this journal \ulcorner

Џll Article views: 4

View related articles $\sqsubset$ 
or else,

\begin{tabular}{l|cccc|c|c}
$r$ & $s$ & $z$ & $\sigma$ & $\zeta$ & $*$ & $*$ \\
$*$ & $*$ & $*$ & $*$ & $*$ & $*$ & $e$ \\
$l$ & $k$ & $g$ & $x$ & $\gamma$ & $y$ & $i$ \\
$m$ & $p$ & $b$ & $f$ & $v$ & $w$ & $u$ \\
$*$ & $*$ & $*$ & $*$ & $*$ & $*$ & $o$ \\
$n$ & $t$ & $d$ & $\boldsymbol{j}$ & $\gamma$ & $*$ & $*$
\end{tabular}

\begin{tabular}{l|llll|l|l}
$n$ & $t$ & $d$ & $p$ & $\gamma$ & $*$ & $*$ \\
$r$ & $s$ & $z$ & $\sigma$ & $\zeta$ & $*$ & $*$ \\
$*$ & $*$ & $*$ & $*$ & $*$ & $*$ & $a$ \\
$l$ & $k$ & $g$ & $x$ & $\gamma$ & $y$ & $i$ \\
$m$ & $p$ & $l$ & $f$ & $v$ & $w$ & $u$ \\
$*$ & $*$ & $*$ & $*$ & $*$ & $*$ & $o$
\end{tabular}

These sequences read both ways, i.e. either horizontally or vertically. The vertical ones, however, are often indistinct, and got at rather by inference and analogy than by the direct testimony of the sounds themselves. Thus the connection between $p$ and $b$ is clearer than that between $m$ and $n$, this latter being clearer than the affinity between $l$ and $m$. The sequence of the vowel, expressed by the horizontal lines, is, however, very distinct.

Such is the amount of sequences which seem to the author sufficiently distinct to claim recognition as the groundwork of phonetic researches. The peculiar transition to the vowels on the part of the sounds of series $k$ and $p$, the affinity with the consonants on the part of the vowels $i$ and $u$, and certain peculiarities of the mutes $s$ and $z$, combined with the rudimentary character of the present arrangement, indicate the probability that further researches will give a more general expression to the affinities in question.

LXI. On the Gas Voltaic Battery.-Experiments made with a view of ascertaining the rationale of its action and its application to Eudiometry. By W. R. Grove, Esq., M.A., F.R.S., Professor of Experimental Philosophy in the London Institution.

(Continued from p. 354 and concluded.)

TT now occurred to me that as several of these gases (take as an instance nitrogen) were absolutely without effect in the gas battery, this would form a valuable instrument for the analysis of atmospheric air or other mixed gases. I therefore procured,

Experiment 24,-Two narrow cubic inch tubes of seven inches long, carefully graduated into 100 parts. These were

* For the power of these signs see Phil. Mag. for February 1841. 
immersed in separate vessels of dilute sulphuric acid, and filled with atmospheric air exactly to the extreme graduation; the water mark within the tube was examined when exactly at the same level as the exterior surface of the liquid; folds of paper were used to protect them from the warmth of the hands, and thus prevent expansion; the barometer and thermometer were examined, and every precaution taken for accurate admeasurement. One of these tubes was left empty in order to ascertain, and eliminate from the result, the effect of solubility. Into the other was placed a strip of platinized platinum foil, one quarter of an inch wide. This strip of foil was connected by a platinum wire with another strip placed in a tube of hydrogen and inserted in the same vessel. The apparatus is shown in fig. 12. After the circuit had been closed for two days, the liquid was found to have risen in the tube $a$ twenty-two parts out of the 100 ; in the tube placed by its side, it had risen one division. The tubes were allowed to remain several day's longer, but no further alteration took place. This analysis gives therefore twenty-one parts in 100 as the amount of oxygen in a given portion of air.

Experiment 25.-The tube $a$ (fig. 12) was charged with nitrogen to a given Fig. 12.

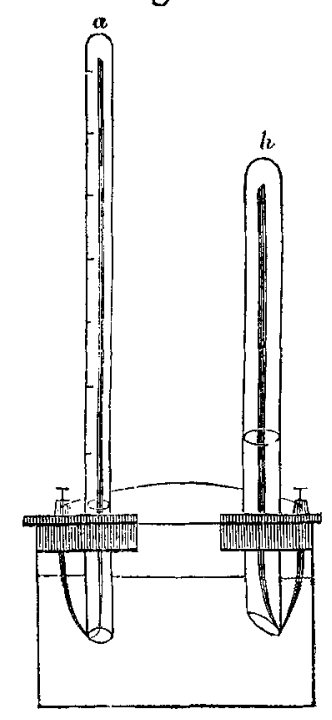
mark, and 0.5 cubic inch of pure hydrogen added, the tube $h$ was then charged with oxygen, and the circuit closed. Examined after twenty-four hours, the water had risen in the tube $a$ exactly 0.5 cubic inch. The apparatus was left in this state for several days, but without any further effect; the voltaic action had thus perfectly exhausted the hydrogen and there. stopped.

These experiments are sufficient to prove the accurate eudiometric action of the gas battery; performed on a large scale this method of eudiometry appears to me likely to possess some advantages. In the eudiometer of Volta, when gases containing oxygen are to be analysed, if the hydrogen added for detonation be impure, the result is of course erroneous. The same may be said of the detonation by spongy platinum, or by a wire heated by a voltaic current, which I formerly proposed *

* Phil. Mag., August 1841, p. 99. 
If, on the other hand, gases containing hydrogen are to be analysed, errors may result from any impurities in the oxygen which is added, or from inaccuracy in the measurement of either gas; in the electrolytic method of eudiometry, the quantity or purity of the hydrogen, in the one case, is of no importance, and in the other, the quantity or purity of the oxygen, that is, provided there be sufficient to exhaust the equivalent to be abstracted from the mixed gas subjected to eudiometry.

It should be observed, that in these experiments only a single pair of the gas battery can be used, as, if more be employed, the electrolyte is likely to be decomposed, and gas added to the compound*. The process is rather slow, but $I$ think very sure. Another valuable application of this process is, that it affords (in experiment 24) a simple method of obtaining nitrogen of unquestionable purity. I know of no method which effects this object so perfectly. All the oxygen of the air is abstracted, as well as that free oxygen which may be contained in the liquid; and by subsequently introducing a little lime-water into the tube $a$, the trifling quantity of carbonic acid may be removed, or the same thing may be at once effected by using caustic potash as the electrolyte in the apparatus fig. 12.

Probably many other applications of the gas battery may suggest themselves to other experimentalists, and obviously many more changes may be rung upon the gases employed, and curious and valuable results obtained; I have, however, in this paper given a sufficient number of experiments fairly to open the subject; each appears so suggestive of new ones that it is difficult to know where to stop.

The experiments on eudiometry, which $I$ have last named, induced me to refer to Dr. Henry's paper on Gaseous Analysist, and on reading it I was struck with a coincidence between the action of spongy platinum on mixed gases and the gas battery, a coincidence strongly confirmatory of the views which led me to its discovery. I will endeavour briefly to state these, and I state them, not as being absolutely correct, for differences of opinion may exist on this as on every other scientific matter, but as being those which existed in my mind prior to the experiments, and which are considerably, and to me unexpectedly, strengthened by the results embodied in the above-mentioned paper of Dr. Henry.

My original deduction may be stated and exemplified as follows:-When pure or amalgamated zinc is immersed in acidulated water, the oxygen, as is well known, will not com-

* See Postscript. + Philosophical Transactions, 1824. 
bine with the zinc; but touch both zinc and liquid with platinum, and combination ensues, the platinum being unaltered. So with a mixture of oxygen and hydrogen, the gases, although in intimate contact, will not chemically unite, but touch them with clean platinum and more or less rapid combination ensues. Here also the platinum is unaltered. Leaving out of the case any purely hypothetical explanation, why may not effects so similar in their character be related in other respects? In the voltaic combination the platinum is heated during action, and if the surfaces, and consequently the quantity of electrochemical action be considerable, it is ignited; so in the catalytic combination, if the platinum be thin and of large extent, or in the form of a sponge, which still more increases its surface, it is ignited. Why, therefore, may we not regard the detonation of gas by platinum as a voltaic effect? or the combination of oxygen and zinc by the presence of platinum a catalytic effect? The only difference is, that gases do not admit of that interchangeable reiation of particles which we call electrolysis. The necessity for this interchange is, however, removed when the gases are in a state of such intimate admixture that it is not requisite to convey the action through a chain of particles in the gas battery this chain is supplied by the intervening electrolyte, and thus the same action which is local in the experiments of Döbereiner is circulating in the gas battery; the latter bears the same relation to the former as the action of the ordinary voltaic battery does to the normal phænomena of chemical affinity. This relation is confirmed by the facts detailed in the paper of Dr. Henry, as the gases which he there found would combine by the presence of spongy platinum, are precisely those which will combine in the gas battery; thus oxygen and hydrogen combine rapidly, oxygen and carbonic oxide much more slowly, and oxygen and olefiant gas very feebly, so much so, as, in Henry's experiments, to require heat to induce combination. Of course chlorine and hydrogen, which will unite without platinum, will, $\alpha$ fortiori, unite with the aid of platinum, or they may in the gas battery occasion secondary action; the oxygen evolved by the decomposition of water by the chlorine combining with the free hydrogen in the tube. As oxygen and ammonia will, when at a slightly elevated temperature, combine by the influence of spongy platinum, forming water and leaving nitrogen, I now, in order further to test this relation, tried

Experiment 26.-Ten cells of the gas battery were charged with oxygen and solution of ammonia, with a little sulphate of ammonia added to improve its conducting power. This arrangement produced a moderate effect upon the iodide, 
which was continuous; the liquid rose slowly but uniformly in the oxygen tubes; a gas was evolved in the alternate tubes, which proved to be pure nitrogen. After three weeks closed circuit, the gases collected, measured, and averaged gave for each tube,

$$
\text { Nitrogen evolved . . }=0.07 \text { cubic inch. }
$$

Oxygen absorbed . . $\cdot=0.12$

Experiment 27.-To examine whether the alkaline character of ammonia had anything to do with the effect, ten cells were charged with oxygen and solution of caustic potash, but produced no effect.

These experiments are strongly corroborative, and seem to me conclusive as to the relation between the action of the gas battery and catalysis by spongy platinum. Experiment 26 is also remarkable in regard to the binary theory of electrolysis, but upon this point I will not here enter.

Applying the hypothesis of Grotthus to the gas battery, we may suppose that when the circuit is completed, at each point of contact of oxygen, water and platinum, in the oxygen tube, a molecule of hydrogen leaves its associated molecule of oxygen to unite with one of the free gas; the oxygen thus thrown off unites with the hydrogen of the adjoining molecule of water; and so on until the last molecule of oxygen unites with a molecule of the free hydrogen; or we may conversely assume that the action commences in the hydrogen tube. In all these cases we should ever bear in mind that we proceed by steps which nature, as hitherto tested by experiment, has not recognised. All we can safely predicate of the actions at anode and cathode is that they are correlations; although they take place at a distance, the one has no more been proved to take place without the other, or before the other, than height has been proved to exist without depth. I therefore allude to this hypothesis, not as literally adhering to it, but because it is generally received, and may tend to associate the action of the gas battery with the ordinary phænomena of electrolysis.

A number of hypotheses has been and may be proposed to account for these and other mysterious phænomenal relations; they all agree in being assimilations of what is unfamiliar to what is familiar. They are undonbtedly useful as didactic illustrations, and it is as such that they have hitherto contributed to advance science. It is, however, a curious circumstance, and worthy of some consideration, that the voltaic hypothesis of Grotthus, the emissive and undulatory hypotheses of light and heat, and, as far as I am aware, all physical hypotheses hitherto propounded, represent natural agencies as effects of motion and matter. These two seem the most di- 
stinct, if not the only conceptions of the mind, with regard to natural phænomena, and when we try to comprehend or explain affections of matter which are not obviously modes of motion, we hypothetically or theoretically reduce them to it: the senses perceive the different effects of sound, light, heat, electricity, \&c., but the mind appears capable of distinctly conceiving them only as modes of motion. Does not this supply an argument that all physical agencies are reducible to these elements of mental conception? Or are we to look for new powers of mind, in other words, will greater familiarity with phænomena, at present recondite, enable the mind more clearly to comprehend them, and avoid the necessity of referring them theoretically to more familiar, and apparently more simple phænomena? To pursue this curious inquiry would involve me in a discussion foreign to the object of this paper and to the general character of contributions to the Royal Society, but the question arises so immediately out of the subject, and is so necessary to explain my own view, that I trust this brief statement of it will be considered sufficiently pertinent. It touches upon that interesting, scarce definable boundary, where physical merges into metaphysical science.

There are one or two other theoretical points as to which the gas battery offers ground of interesting speculation; the contact theory is one. If mysnotion of that theory be correct, I am at a loss to know how the action of this battery will be found consistent with it. If, indeed, the contact theory assume contact as the efficient cause of voltaic action, but admit that this can only be circulated by chemical action, I see little difference, save in the mere hypothetical expression, between the contact and chemical theories; any conclusion which would flow from the one would likewise be deducible from the other; there is no sequence of time in the phænomena, the contact or completion of the circuit and the electrolytical action are synchronous. If this be the view of contact theorists, the rival theories are mere disputes about terms. If, however, the contact theory connects with the term contact an idea of force which does or may produce a voltaic current independently of chemical action, a force without consumption, I cannot but regard it as inconsistent with the whole tenor of voltaic facts and general experience.

Another point of theory suggested by the gas battery, is the relation of latent heat in the different cells of the battery and voltameter. According to our received theory of caloric, oxygen and hydrogen cannot assume the gaseous from the liquid state without rendering sensible heat latent. Now, as in the gas battery the gases evolved from the liquid in the 
voltameter must require and absorb precisely as much heat as is set free by the gases becoming liquid in each cell, it may be a curious subject of future inquiry (an inquiry which that beautiful instrument, the thermo-multiplier, will materially aid) to ascertain whether the heat absorbed in the voltameter be exacted from surrounding bodies, or whether it be supplied by the action of the battery itself, $i$. e. as the chemical force in the voltameter is conversely equivalent to that in each cell of the battery, and the calorific force at the voltameter is also the converse equivalent of that in each battery cell, whether there is the same mutual dependence of the latter as of the former forces. 'The action in the voltameter of ordinary batteries would argue strongly against the proposition, that the heat is exacted from surrounding bodies, as it is well known that water when electrolysed has its temperature rather increased than diminished; and I have found, when decomposing water with the nitric acid battery at a rate of 150 cubic inches a minute, a very considerable augmentation of temperature in the liquid subjected to decomposition, so much so, that if the quantity was not considerable, it was heated to ebullition. Much of this adventitious heat may have arisen from the restriction of the circuit by the voltameter plates and connecting wires, but if the gas battery be supposed to supply exactly sufficient heat, or (to use a license of expression) to convert electricity into sufficient heat to satisfy the demands of the expanding gases, - each battery cell being able by the condensation of its respective gases to afford this supply, $-a$ rise of temperature ought to be perceptible in the whole battery equal to the heat produced by the condensation of gases in all the cells, minus that of one cell. I have not as yet been able to detect any elevation of temperature due to the action of the gas battery, not having in my possession any instrument capable of detecting such delicate thermoscopic effects. I am, therefore, the more anxious to offer the point for the consideration of those who may have such instruments at their command; and here for the present I leave the gas battery and its theory.

London Institution, March 12, 1843.

$$
\text { Postscript, July } 7 \text { th. }
$$

The length of time which has elapsed between the communication and printing of this paper, as it has enabled me to procure the apparatus fig. 8, will I trust be deemed a sufficient reason for my adding a Postscript containing a few experiments with this form of battery, some of which I cannot but consider important. 
Experiment 28.-In order further to test the opinion expressed, p. 423, six cells of this battery were charged with pure hydrogen and dilute acid in the alternate tubes. When first charged they decomposed water freely; but after the circuit had been closed for a short time, to exhaust the oxygen of the atmospheric air in solution, they produced no voltaic effect; the whole series of six would not decompose iodide of potassium; when, however, a little air was allowed to enter any one of the tubes containing liquid, that single cell instantly decomposed the iodide; three cells were put aside, each in closed circuit; at the expiration of a week these produced no effect upon a galvanometer, nor was there any gas evolved in the tubes containing liquid; the stoppers were now taken out, and the liquid in the hydrogen tubes rose to an average of 0.3 cubic inch; each cell contained a pint, and we may therefore regard 0.15 cubic inch as the amount of oxygen held in solution by this quantity of acidulated water. Were it not for the extreme practical difficulty of perfectly excluding atmospheric air for a long period, the above would furnish an excellent method of examining the quantity of oxygen held in solution by water, and by applying the proper calculus we might read off on our galvanometer scale the infinitesimal bubbles of gas contained in a given bulk of liquid; if, however, the acid water or the hydrogen contain foreign ingredients, a very different result follows, and the liquid, for reasons which will now be obvious, frequently rises considerably in the hydrogen tubes.

Experiment 29.-I repeated experiment 24, with the battery fig. 8, expecting that as the external air was shut out I should obtain the result more speedily; I was indeed not without a vague hope of producing some effect upon the nitrogen. The first result did follow; upon taking out the stoppers the morning after the battery had been charged, the liquid rose in the air-tube one-fifth of the gaseous volume. I now closed it again and examined it three days afterwards; a very curious effect had taken place; the volume of the gas in the air-tube which had previously contracted had now increased, and it continued slowly increasing day after day. I at first believed that the nitrogen was decomposed, but after many conjectures and experiments found that the increase was due to the addition of hydrogen, a fact to me more extraordinary than the decomposition of nitrogen would have been. On repeating the experiment with nitrogen instead of air the same effect took place, but of course without the previous contraction. I now returned to battery fig. 4 ; several of these cells charged, some with atmospheric air and hydrogen, and others with ni- 
trogen and hydrogen, did not exhibit the effect, though suffered to remain six weeks, each in closed circuit.

To ascertain whether the vacuum formed by the abstraction of oxygen from the liquid had anything to do with the above effect, a central narrow tube, open at both ends, was substituted for the stopper in the battery fig. 8 ; the hydrogen was still evolved. Not to detail a tedious set of test experiments, $I$ at length found that two points were essential to obtaining the effect with certainty ; first, the exclusion of any notable quantity of atmospheric air from solution; and secondly, great purity in the hydrogen. In the former case, when the hydrogen could find oxygen to combine with, it was not evolved; in the latter, there would be mixed or rather diluted gas on both sides, and the forces would be balanced; thus I have never succeeded in obtaining the effect in the open battery, fig. 4, with hydrogen obtained in the ordinary way from granulated zinc or iron filings, but have sometimes succeeded with hydrogen procured by electrolysis. In the battery fig. 8, I have succeeded in producing the effect, but in a feeble degree, from hydrogen obtained in the common way, but have never failed with hydrogen obtained by electrolysis. Oxygen of the greatest purity, voltaically associated with nitrogen, does not produce a similar effect. The above unexpected results render it necessary, in order to ensure accuracy in the eudiometric experiment 24, either purposely to use common hydrogen in the batteries figs. $\$$ and 12 , or, what is more expeditious and accurate, to use a battery similar to fig. 8 , but with tubes longer in proportion to their width; and having first charged the tubes with hydrogen and atmospheric air, to allow these to remain in closed circuit until all the oxygen is abstracted and a little hydrogen added, by the electrolytic effect, to the residual nitrogen; then to substitute oxygen for the original hydrogen, which will in its turn abstract the hydrogen from the nitrogen and leave only pure nitrogen. I have frequently done this with perfect success.

Experiment 30.- Hydrogen and carbonic acid in battery fig. 8 produced the same effect. The volume of the carbonic acid was increased, and hydrogen was found to have been added to it. The effect therefore is not due to any peculiarity of nitrogen, but yet some gas is necessary, for experiment 28 proves that hydrogen alone will not decompose water. I need scarcely say, that when the above-mentioned effect took place an interposed galvanometer was deflected, but the current was much too feeble to decompose iodide of potassium.

I have tried, associated with hydrogen in battery fig. 8, carbonic oxide, olefiant gas, protoxide of nitrogen, and deut- 
oxide of nitrogen; the two former produced no current or chemical effect, the two latter gave a current and were decomposed. The volume of the deutoxide contracted one-half, this was found to be nitrogen, which thenceforth was gradually increased by hydrogen. The volume of the protoxide did not undergo the previous contraction, except slightly from solubility, but its change of state was denoted by the absorption of hydrogen in the associated tube.

I likewise tried the effect of a vacuum and hydrogen, by charging a battery (fig. 8) with 1 cubic inch oxygen and 3 cubic inches hydrogen; the current was much enfeebled by the resistance offered by the vacuum, at first iodide of potassium was decomposed and the galvanometer needle whirled round. After twenty-four hours the galvanometer needle was only deflected $10^{\circ}$, thus a physical was opposed to, and resisted, a chemical force; the current however continued, and all the gas in the oxygen tube disappeared, except a minute bubble; this was probably nitrogen from the atmospheric air in solution, which had escaped to fill the vacuum. When the stopper was taken out the liquid rose suddenly in the hydrogen tube $2 \cdot 2$ cubic inches, giving the equivalent of the oxygen in the tube and in solution. It is very possible that this experiment repeated might sometimes exhibit an evolution of hydrogen in the oxygen tube arising from the escape of the nitrogen of the atmospheric air in solution, and acting as in experiment 29 , but I have not seen this effect take place. It should be distinctly understood, that in all the experiments mentioned in this Postscript, except the first part of experiment 28 , single cells only were used.

Upon the theory of the experiments 29 and 30 I will venture no positive opinion. That gaseous hydrogen should abstract oxygen from hydrogen, without the latter forming any other combination, is a fact so novel, that any attempted explanation is likely to prove premature. If, contrary to the views of Dalton, we suppose that gases when mixed are held together by a feeble chemical affinity, then we may say that the affinity of the nitrogen or carbonic acid for hydrogen produces the effect; the affinity of the oxygen of the water, being balanced between the hydrogen in the liquid and that in the tube, would enable the resultant feeble affinity of the nitrogen for hydrogen to prevail ; but on this supposition, why does not oxygen produce an analogous effect? Its tendency directly to combine with platinum may indeed be regarded as an opposing force, but this tendency is by many considered hypothetical. On the other hand, it may be called an effect of contact; but this, unconnected with a chemical theory, 
presents no other idea to the mind than the fact itself presents, it furnishes no link by which we may extend the phænomena. I therefore, until a better theory be found, should be inclined to adopt the former view, and to regard mixed gas as in a state of feeble chemical union, the more especially as throughout nature we find no absolute lines of demarcation, though for conventional reasons we are obliged to adopt them; there must be many cases in which it is difficult, if not impossible, to draw the line between mechanical mixture and chemical combination.

In conclusion, I would say with regard to the whole of the experiments contained in this paper, that a longer time and more experience may give positive results in cases where I have only obtained negative ones; it is far from impossible that since curious solid combinations are formed by slow electrical currents, as in the experiments of Crosse and Becquerel, so novel gaseous or liquid products may be obtained by the long-continued voltaic action of gases and liquids. This time alone can show.

For previous experiments and theories on the combination of gases by platinum, I may refer to Döbereiner's paper, Phil. Mag. Oct. 1823, in which I find he expresses an opinion that it is a voltaic effect; to the papers of Dulong and Thenard, Annales de Chimie, tom. 23 and 24; and to Faraday's Experimental Researches, Series 6. The various experiments on polarized electrodes of Ritter, Faraday, De la Rive, Becquerel, Matteucci, and Schonbein, are also in point.

LXII. On the Form of Equilibrium of an Inextensible String laid on a surface and acted on by any forces. By the Rev. J. A. Coombe, M.A., Fellow of St. John's College, Cambridge*.

To the Editors of the Philosophical Magazine and Journal. GentLemen,

$7 \mathrm{HE}$ following method of investigating the form of equi1 librium of an inextensible string laid on a surface and acted on by any forces, has the advantage of presenting a result extremely neat and symmetrical. I adhere to the suffix notation in expressing a differential coefficient, never having yet seen any arguments, of much value, brought against this elegant and convenient method.

Let $u=0$ be the equation to the surface.

$x y z$ the rectangular coordinates of any point of the string, and therefore of a point in the surface.

* Communicated by the Author. 